\title{
Spatial Distribution of Dengue Incidence: A Case Study in Putrajaya
}

\author{
Mohd Hazrin', Helen Guat Hiong1, Nadzri Jai' ${ }^{1}$, Norzawati Yeop', Muhammad Hatta², \\ Faizah Paiwai', S. Joanita ${ }^{3}$, W. Othman ${ }^{4}$ \\ ${ }^{1}$ Institute for Public Health, Ministry of Health Malaysia, Kuala Lumpur, Malaysia \\ ${ }^{2}$ Federal Health Department of Kuala Lumpur, Kuala Lumpur, Malaysia \\ ${ }^{3}$ Hospital Sultanah Ampuan Rahimah, Klang, Malaysia \\ ${ }^{4}$ District Health Office of Putrajaya, Putrajaya, Malaysia \\ Email: hazrin@moh.gov.my
}

Received 25 November 2015; accepted 21 February 2016; published 24 February 2016

Copyright (C) 2016 by authors and Scientific Research Publishing Inc.

This work is licensed under the Creative Commons Attribution International License (CC BY).

http://creativecommons.org/licenses/by/4.0/

c) (i) Open Access

\section{Abstract}

Background: Dengue is a viral disease transmitted by Aedes aegypti mosquito. Dengue has become an important public health problem worldwide. It affects tropical and subtropical regions around the world, predominantly in urban and semi urban areas. Dengue outbreaks characteristically have been associated with high rainfall as well as elevated temperatures and humidity. In Malaysia, dengue fever (DF) and dengue haemorrhagic fever (DHF) have shown an increasing trend. This study aimed to map the spatial distributions of dengue cases in Putrajaya through integration of Geographical Information System (GIS) and spatial statistical analyses. Methodology: This study analysed 389 dengue cases from 2013 to 2014 in different precincts in Putrajaya. Data were collected from various government health agencies. Three spatial statistical analyses [Moran's I, Average Nearest Neighbourhood (ANN) and Kernel Density estimation] were used to access spatial distribution cases. Results: Analysis showed dengue cases within the district were highly clustered and occurred at an average distance of $\mathbf{2 6 4 . 9 1}$ meters. Several locations especially residential areas had been identified as hot spots of dengue cases in the precinct by using kernel density estimation analysis. Conclusion: The study has shown that by integrating spatial analysis using GIS, it is possible to improve the understanding of the distribution of dengue cases within a particular area. GIS and spatial statistical analyses are important in guiding health agencies, epidemiologists, public health officers, town planners and relevant authorities in developing efficient control measures and contingency programmes in identifying and prioritizing their efforts in effective dengue control activities.

\section{Keywords}

Dengue, Incidence, GIS, Spatial Analysis, Malaysia 


\section{Introduction}

Dengue is an illness caused by a virus that is spread through mosquito bites. Symptoms include fever, headache, nausea, vomiting, rash, and pain in the eyes, joints, and muscles [1]. With more than one-third of the world's population living in areas at risk for infection, dengue virus is a leading cause of illness and death in the tropics and subtropics. There is no specific treatment for dengue, however, with frequent and appropriate medical care; the lives of patients with the more serious dengue haemorrhagic fever (DHF) could be saved. The only way to prevent the transmission of dengue virus is to combat the carrier [2].

Dengue infection is caused by dengue virus which is a mosquito-borne flavivirus. It is transmitted by Aedes aegypti and Aedes albopictus. There are four distinct serotypes, DEN-1, 2, 3 and 4. Each episode of infection induces a life-long protective immunity to the homologous serotype but confers only partial and transient protection against subsequent infection by the other three serotypes [2].

According to World Health Organization, more than 108,698 dengue cases, including 215 deaths have been reported in Malaysia in 2014. This is a 151\% increase in cases in 2014 compared with the same period in 2013 [3]. The number of reported dengue fever (DF) cases in Malaysia had shown an increasing trend from 20042014 (Figure 1).

The incidence rate had also shown an upward trend from 132.5 cases/100,000 population in 2004 to 343.1 cases/100,000 population in 2014 (Figure 2). This exceeds the national target for the incidence rate of DF and

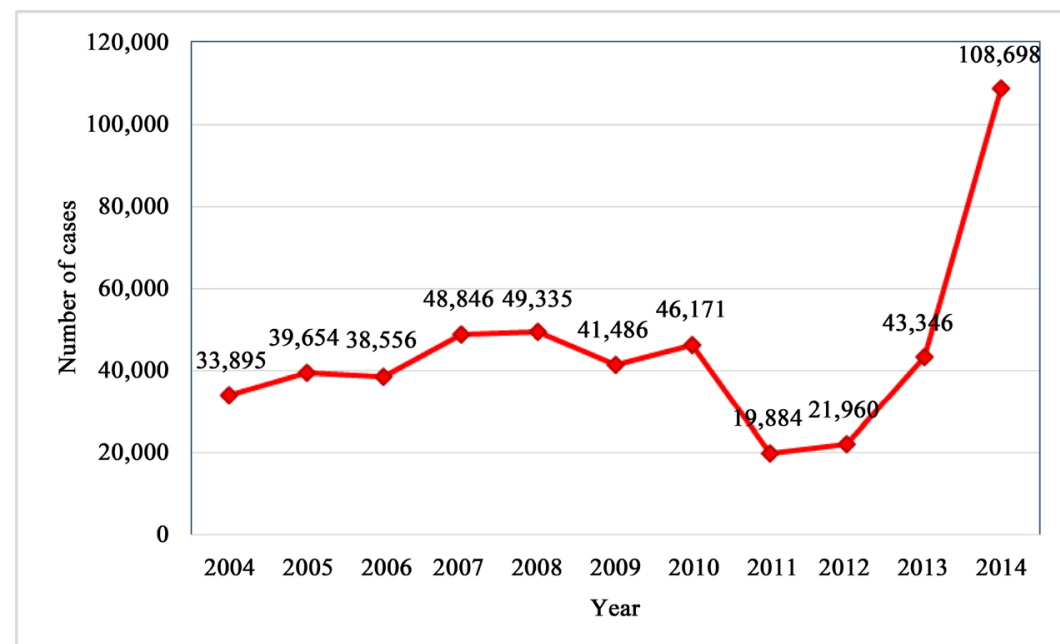

Figure 1. Number of dengue cases, Malaysia 2004-2014.

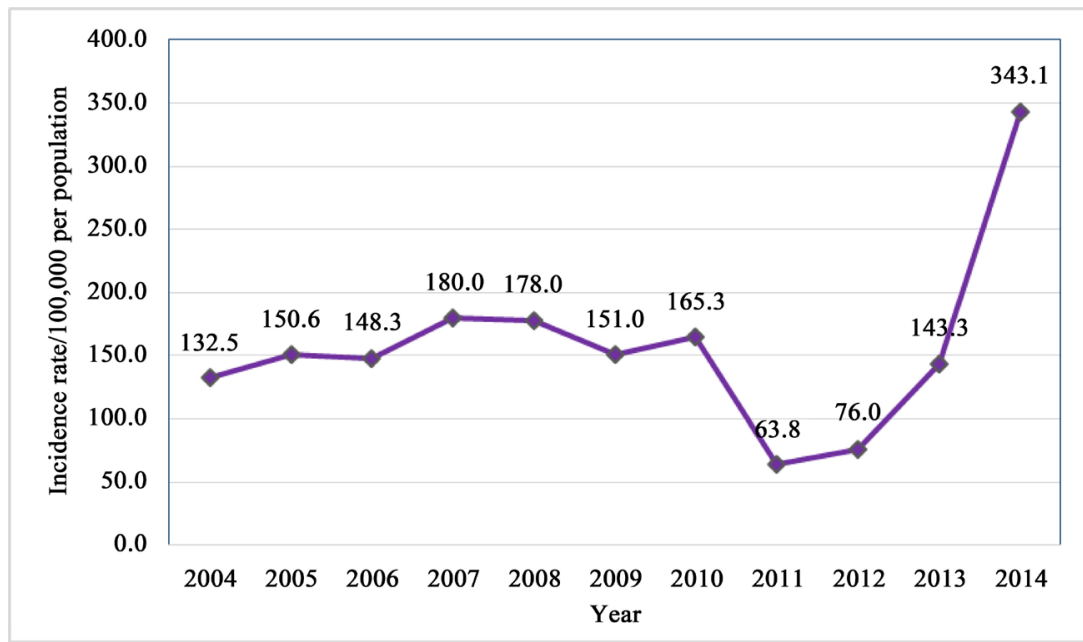

Figure 2. Dengue incidence rate by age group in Malaysia, 2004-2014. 
DHF which is less than 50 cases/100,000 population. Dengue fever accounted for almost $95 \%$ of all reported cases. The serologically confirmed cases were approximately $40 \%-50 \%$ of these cases at the time of notification [2].

The utilisation of geographical information system (GIS), a computerized system that combines spatial and descriptive data for mapping and analysis is being increasingly used to collate and map available epidemiological information and relate it to factors such as climate and environmental factors known to influence distribution of diseases together with time, people and other dimension of interest [4].

GIS plays an important role in disease surveillance and control of the mosquito-borne diseases as they assist in the analysis of potential risk factors associated with the disease through the geo-coding processes [5] and facilitate maps that are useful for the identification of spatially and temporally localised areas of potential high-risk populations [6]-[10]. The visualised information represented in different types of maps based on GIS enables simultaneous observation of both attribute and geographical relationships [11]-[13]. Maps also help policy decision-makers and public health officials to communicate with the public and policy decision-makers about complex information in an easily interpretable format [13] [14].

GIS can provide not only an opportunity to improve our understanding of the distribution patterns of dengue, but can also provide an environmentally and socially informed platform to develop the elements of an early warning system towards control and prevention of dengue [8]. The objective of this study was to analyze the dengue outbreak pattern in Putrajaya in terms of their spatial dissemination and hotspot identification.

\section{Methods}

\subsection{Study Area}

Putrajaya located in $25 \mathrm{~km}$ southern Kuala Lumpur, is the federal administrative capital of Malaysia. Putrajaya was used as our study area because it was increasing trend of dengue cases from 2012 to 2014. This study used precinct, the smallest local governing unit in Malaysia, as the spatial zoning boundary. All of the precinct were under jurisdiction of Perbadanan Putrajaya (Figure 3).

\subsection{Data Collection}

Several types of data were incorporated into GIS environment such as dengue case data and demographic data. These data were gathered from various sources.

\subsection{Dengue Data}

Dengue data from years 2012 until 2014 were obtained from Putrajaya District Health Office. All data were sorted according to each precinct and years. All the cases were documented in e-dengue database and extracted to Microsoft Excel program. Figure 4 shows the number of dengue cases for each precinct in Putrajaya. Attributes such as gender, age, ethicity, occupation, address, date of admission and date of discharge, type of dengue, locality of dengue incidence and number of weeks of dengue cases. During the data smoothing process, we excluded the uncompleted data to avoid any biasness. In order to synchronize all digital data coordinate system, we decided to use World Geodetic System (WGS 84) which serve the x, y of an object by longitude and latitude respectively. The coordinate or point of the dengue incidence was essential in this study as it reflect the spatial correlation of dengue between each sub-district.

\subsection{Spatial Analysis}

In this study, the spatial distribution of dengue incidence within Putrajaya area was examined using spatial statistic method. First, a global spatial autocorrelation method which is Moran's I was used to evaluate whether the pattern expressed was clustered, dispersed or random. The tool calculates the Moran's I Index value and both $\mathrm{z}$-score and p-value to evaluate the significance of that Index. P-values are numerical approximations of the area under the curve for a known distribution, limited by the test statistic.

The Moran's I statistic for spatial autocorrelation given as:

$$
I=\frac{n \sum \sum_{1}^{n} W_{i j}\left(x_{i}-\bar{x}\right)\left(x_{j}-\bar{x}\right)}{W \sum\left(x_{i}-\bar{x}\right)^{2}}
$$






Figure 3. Map of Putrajaya. 


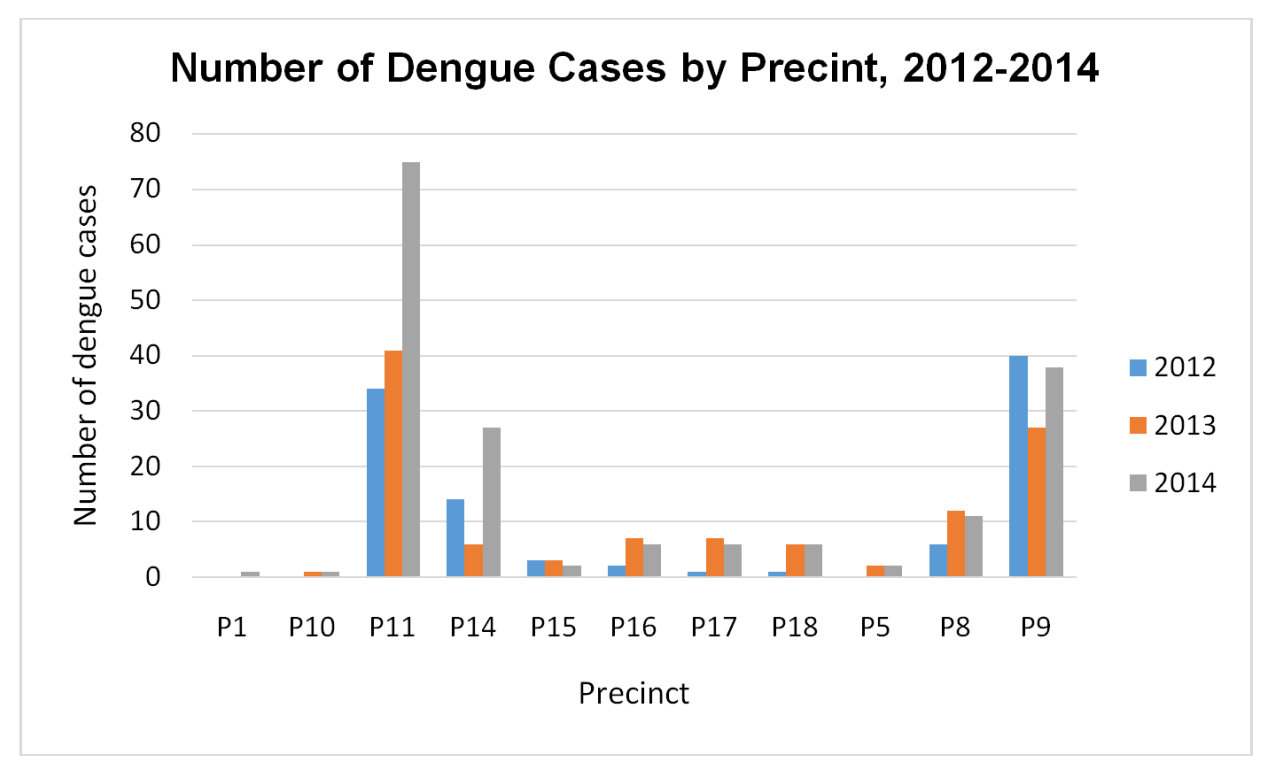

Figure 4. Number of reported dengue cases for each precinct in Putrajaya for years 2012-2014.

where $x$ is the number of cases, $x$ is the mean of the variable, $x_{i}$ is the variable value at a particular location $i, x_{j}$ is the variable value at another location $j$, and $W_{i j}$ is a weight indexing location of $i$ relative to $j$. The value of Moran's I range from -1 for strong negative spatial autocorrelation to +1 for strong positive spatial autocorrelation. A value near 0 would indicate a spatially random pattern.

The Average Nearest Neighbor (ANN) tool measures the distance between each feature centroid and its nearest neighbor's centroid location and averages all these nearest neighbor distances. If the average distance is less than the average for a hypothetical random distribution, the distribution of the features being analyzed is considered clustered. If the average distance is greater than a hypothetical random distribution, the features are considered dispersed. The average nearest neighbor ratio is calculated as the observed average distance divided by the expected average distance (with expected average distance being based on a hypothetical random distribution with the same number of features covering the same total area).

The average Nearest Neighbour is given as:

$$
A N N=\frac{\bar{D}_{O}}{\bar{D}_{E}}
$$

where $D_{O}$ is the observed mean distance between each feature and their nearest neighbour:

$$
\bar{D}_{O}=\frac{\sum_{i=1}^{n} d_{i}}{n}
$$

And $D_{E}$ is the expected mean distance for the features given a random pattern:

$$
\bar{D}_{E}=\frac{0.5}{\sqrt{n / A}}
$$

In the above equations, $d_{i}$ equals the distance between feature $i$ and its nearest feature, $n$ corresponds to the total number of features and $A$ is the total study area. The $Z_{A N N}$ score for the statistic is calculated as:

$$
z_{\text {ANN }}=\frac{\bar{D}_{O}-\bar{D}_{E}}{S E}
$$

where:

$$
S E=\frac{0.26136}{\sqrt{n^{2} / A}}
$$


Hot spot analysis using Kernel density estimation interpolation technique was used to calculate the density of point features around each output raster cell. According to Keim, kernel density estimation is an effective tool to identify high-risk areas within point patterns of disease incidence by producing a smooth, continuous surface that defines the level of risk for that area [13]. Kernel density estimation is an interpolation that is appropriate for individual point locations [12]. It calculates the density of point features around each output raster cell. According to Indaratna, the kernel estimate is a better 'hotspot' identifier than the cluster analysis [12]. Kernel density estimation is a useful method as it helps to precisely identify the location, spatial extent and intensity of dengue disease hotspots.

\section{Results}

A total of 389 dengue cases were reported from 2012 to 2014 in Putrajaya with Precinct 11 (150 cases) notified the highest number of cases followed by Precinct 9 (105 cases), Precinct 14 (47 cases), Precinct 8 (29 cases), Precinct 16 (15 cases), Precinct 17 (14 cases), Precinct 18 (13 cases), Precinct 15 (8 cases), and Precinct 5 (4 cases), Precinct 10 (2 cases) while both Precinct 1 recorded the lowest cases with only one case.

\subsection{Spatial Distribution}

Moran's I was used as an index of spatial autocorrelation to access the spatial distribution of dengue cases within Putrajaya. Result from Moran's I test on dengue incidence for Putrajaya indicated that there was positive spatial autocorrelation among dengue incidence within precincts. The Moran's I for dengue cases is -0.05 ( $\mathrm{p}<$ 0.01 ) while the z-score is 0.05 ( $p<0.01)$ (Figure 5).

\subsection{Distance Analysis}

The result of ANN analysis gives three values which are Nearest Neighbour Ratio (R), z-scores and p-value. It was found from analysis the distribution of dengue cases in the Putrajaya for 2013 and 2014 was spatially clustered. Results from ANN analysis showed that the average nearest neighbour ratio was less than 1; i.e. 0.37 ( $\mathrm{p}<$ 0.0001). Figure 6 shows the cluster pattern of dengue cases in Putrajaya district. The z-score for dengue incidence within the Precincts was $-16.44(\mathrm{p}<0.0001)$ and the significant spatial autocorrelation of dengue incidences occurred at an average distance of 264.91 meters.

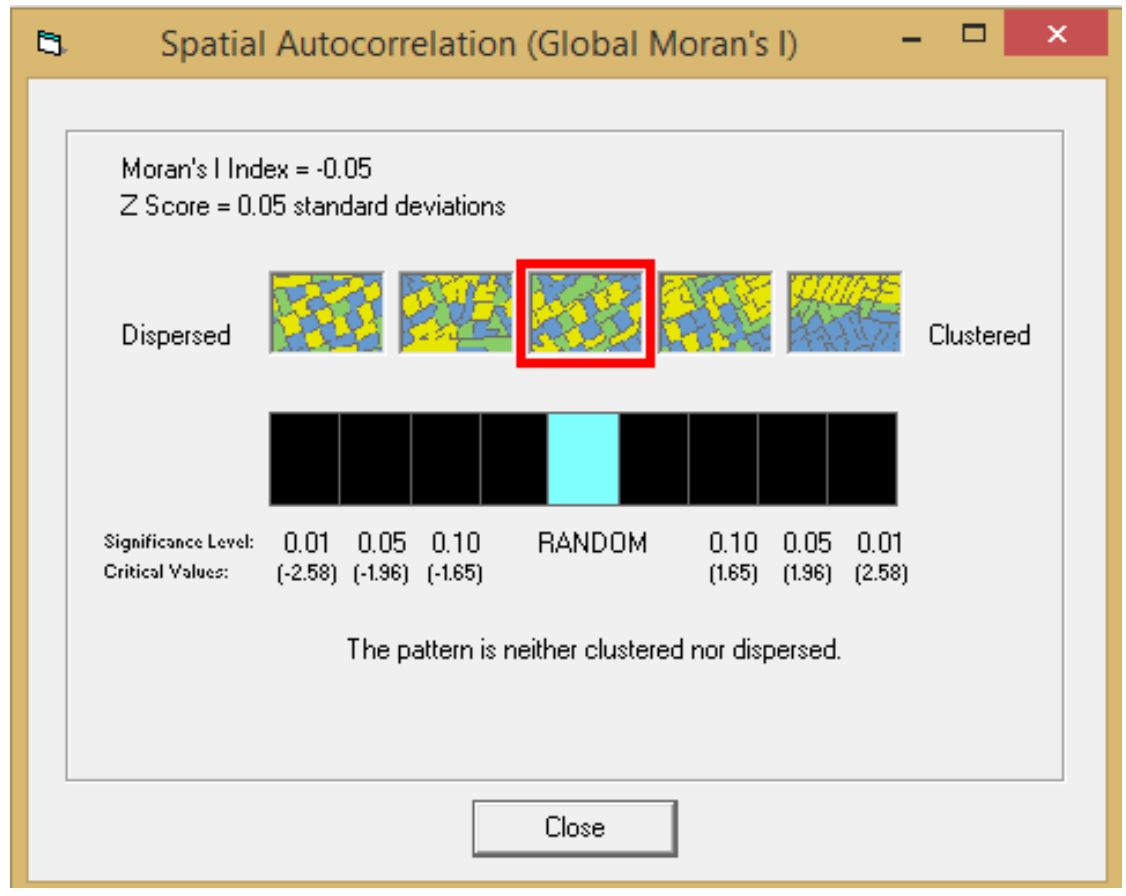

Figure 5. Result of global Moran’s I for dengue cases within Putrajaya. 


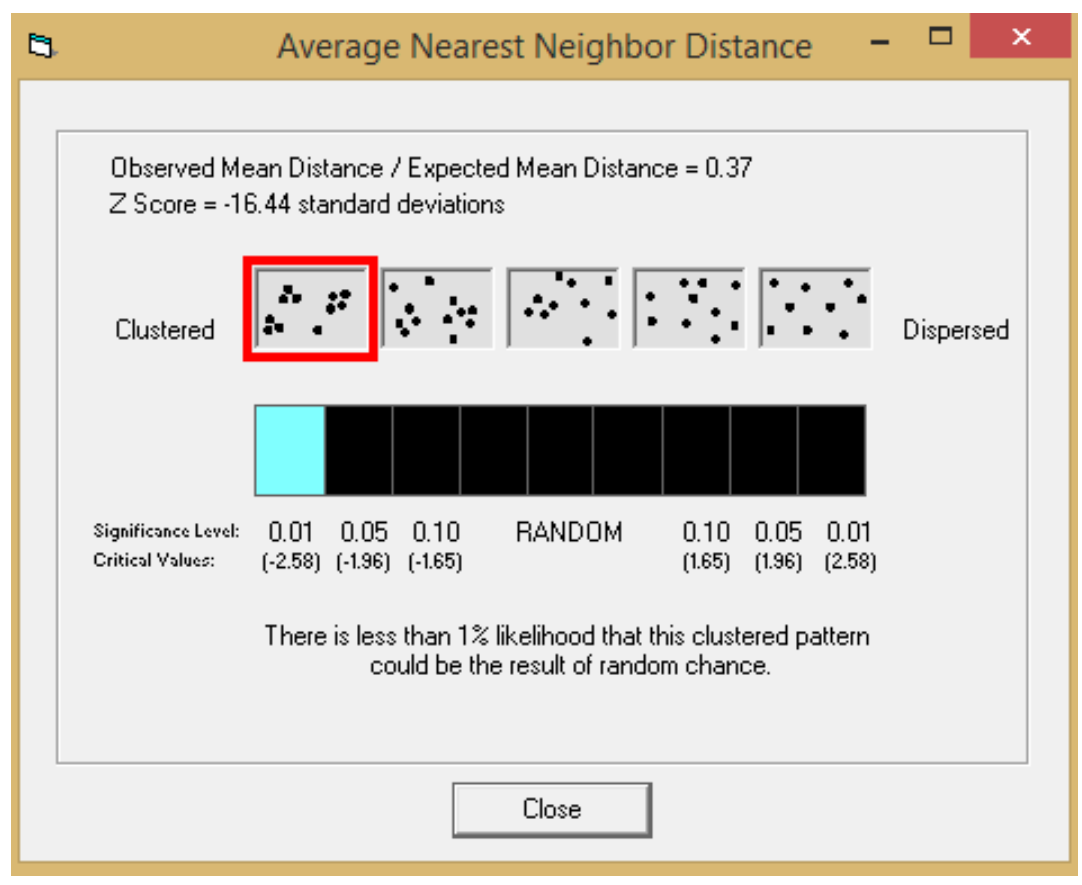

Figure 6. Average Nearest Neighbour statistic result.

\subsection{Hot Spot}

Kernel density estimation was applied in this study to locate 'hot spot' for dengue cases in Putrajaya District. The dark colour area in Figure 7 is the hotspot identified with maximum dengue incidence density. Hence, with the help of dengue density map we are able to target specific area within plot showing highest incidence.

Overall picture of dengue density variation within the plots can be known with the help of kernel density map. From the result, we can identify the area most affected by dengue disease. Generally, most of the hot spot locations were located in Precinct 11. Hot spot locations were also found at Precinct 14, Precinct 9, Precinct 7, Precinct 8, Precinct 16, Precinct 17 and Precinct 18. All of the detected hot spots were situated at residential areas especially apartments.

\section{Discussion}

Our study found out that the dengue cases in the precincts were spatially autocorrelated through Moran's I indices. The dengue cases were distributed at random chances. These findings were different with Er's study in Hulu Langat district where spatial distribution were spatially clustered [14]. However, ANN analysis stated that the dengue cases were highly clustered and it was found that the significant spatial autocorrelation of dengue incidences occurred at an average distance of 264.91 meters. This could be due to the fact that spatial clustering of disease is almost inevitable since human population generally live in spatial clusters rather than random distribution in space [15]. Previous studies in Kuala Lumpur city [14] and Hulu Langat district [16] also indicated spatial clustered patterns of dengue cases.

Kernel density estimation was a useful tool to locate the "hot spot" whereby traditional use of incidence data cannot distinguish the spatial differences in risk areas from those of their neighbouring areas, which is crucial for the effective control of vector-borne infectious diseases [17]. It was proven from this study that by using GIS and spatial statistic tools, we can determine the spatial autocorrelation between dengue cases and population.

Data on disease vectors is important because human-mosquito contact is the main risk factor of dengue fever, thus additional data on the principal mosquito vector, such as the number of containers or control measures of breeding sites would give an estimate of vector presence [17]. Future research should extend from current study to a national scale analysing with more rigorous geo-statistical analysis tools to avoid oversimplification of climatological impact on dengue transmission. Better understanding of the mosquito's gonotrophic cycle and virus latency period may also be crucial in the planning of an efficient dengue control programme [18]. 


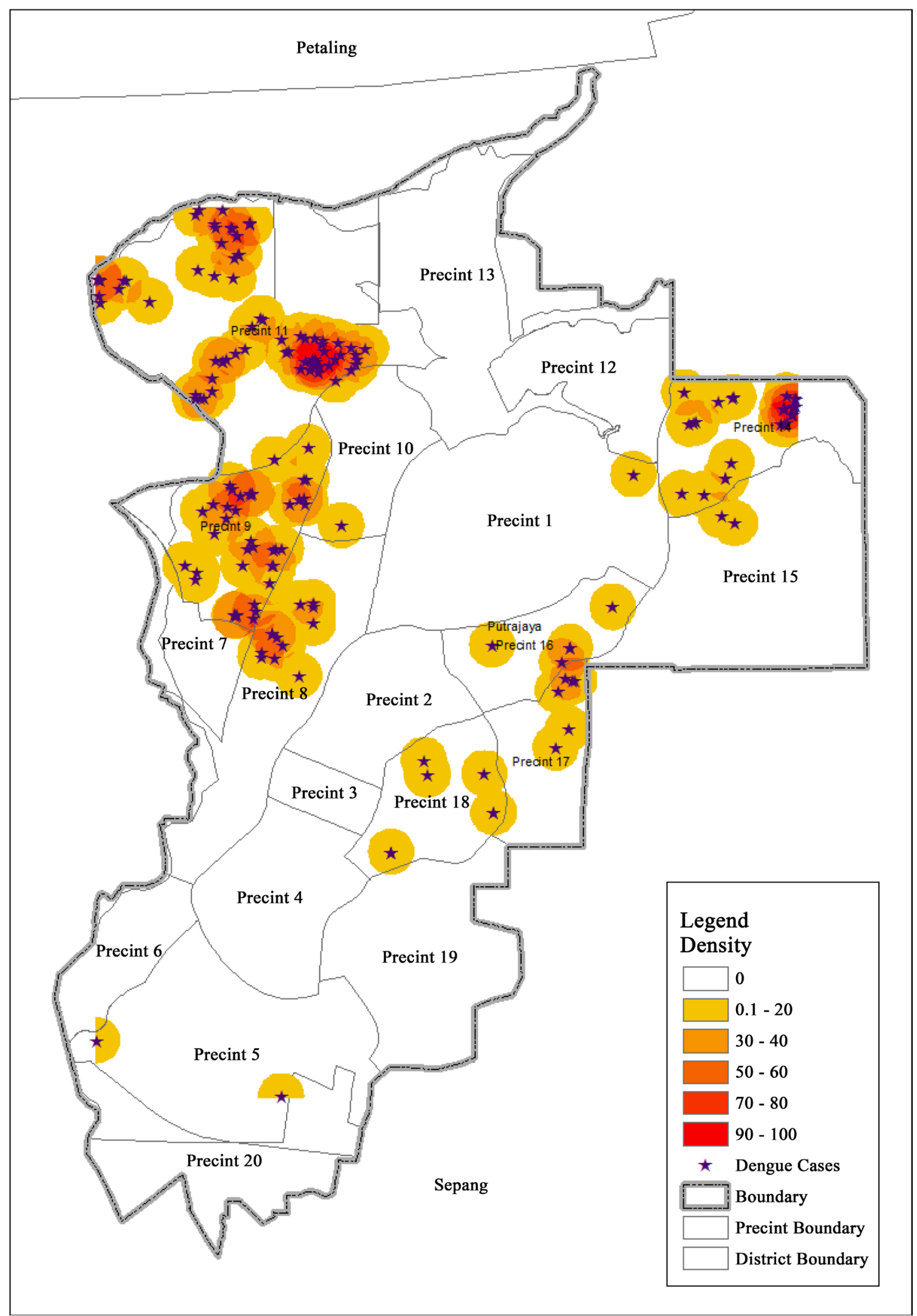

Figure 7. Hot spot locations of dengue cases in Putrajaya. 
Activities for the prevention and control of dengue infection carried out in Malaysia should consider to incorporate analysis of environmental factors, thus this study analysed the spatial distribution of dengue cases in the Federal Territory of Putrajaya. The study was done by coupling the data manipulation and geographic presentation capability of GIS and spatial statistic to map the spatial distribution of dengue cases. Furthermore, this study provided a new dimension to the health authorities in Malaysia, specifically in the potential of using GIS application to develop strategies for the implementation of preventive and control activities for dengue infection.

\section{Conclusion}

The study has shown that by integrating spatial analysis using GIS; it is possible to improve the understanding of the distribution of dengue cases within a particular area. This study has proven that it is possible to improve the understanding of dengue cases distribution within areas without relying on information about the density of mosquitoes. GIS and spatial statistical analyses are important in guiding district health office of Putrajaya and Perbadanan Putrajaya in developing efficient control measures and contingency programmes in identifying and prioritizing their efforts in effective dengue control activities.

\section{Acknowledgements}

We would like to thank the Director General of Health Malaysia for his kind permission to publish this paper. We would like to thank the Putrajaya District Health Office for providing us with the data.

\section{References}

[1] What Is Dengue? Centers for Disease Control and Prevention WHO. http://wwwnc.cdc.gov/travel/diseases/dengue

[2] Medical Development Division, Ministry of Health Malaysia. Management of Dengue Infection Adults. 2014.

[3] Number of Reported Cases of Dengue Fever and Dengue Hemorrhagic Fever (DF/DHF) in the Western Pacific Region, by Country. http://www.wpro.who.int/emerging_diseases/annual.dengue.data.wpr/en/

[4] Burrough, P.A. and McDonnell, R.A. (1998) Principals of Geographical Information Systems. Oxford University Press, Oxford.

[5] Lloyd, C. (2010) Spatial Data Analysis-An Introduction for GIS Users. Chapter 2, Oxford University Press, Oxford.

[6] Fotheringham, A.S. and Rogerson, P.A., Eds. (2009) The Sage Handbook of Spatial Analysis. Sage Publications, London, 183-206.

[7] Lloyd, C. (2010) Spatial Data Analysis-An Introduction for GIS Users. Chapter 4, Oxford University Press, Oxford.

[8] Collinge, S.K. (2010) Spatial Ecology and Conservation. Nature, 1, 69.

[9] Haining, R. (2003) Spatial Data Analysis: Theory and Practice. Cambridge University Press, Cambridge. http://dx.doi.org/10.1017/CBO9780511754944

[10] Githeko, A.K. (2012) Advances in Developing a Climate Based Dengue Outbreak Models in Dhaka, Bangladesh: Challenges \& Opportunities. Indian Journal of Medical Research, 136, 7-9.

[11] Cleveland, W.S. (1993) Visualising Data. Hobart Press, Summit.

[12] Indaratna, K., Hutubessy, R., Chupraphawan, S., Sukapurana, C., Tao, J., et al. (1998) Application of Geographical Information Systems to Co-Analysis of Disease and Economic Resources: Dengue and Malaria in Thailand. The Southeast Asian Journal of Tropical Medicine and Public Health, 29, 669-684.

[13] Kitron, U. (2000) Risk Maps: Transmission and Burden of Vector-Borne Diseases. Parasitoly Today, 16, $324-325$. http://dx.doi.org/10.1016/S0169-4758(00)01708-7

[14] Rezaeian, M. (2008) How to Visualize Public Health Data? Part Two: Direct and Indirect Standardization Methods. Middle East Journal of Family Medicine, 7, 42-44.

[15] Keim, D.A. (2002) Information Visualization and Visual Data Mining. IEEE Transactions on Visualization and Computer Graphics, 8, 1-8. http://dx.doi.org/10.1109/2945.981847

[16] Er, A.C., Rosli, M.H., Asmahani, A., Mohamad Naim, M.R. and Harsuzilawati, M. (2010) Spatial Mapping of Dengue Incidence: A Case Study in Hulu Langat District, Selangor, Malaysia. International Journal of Environmental, Chemical, Ecological, Geological and Geophysical Engineering, 4, 251-255.

[17] Lawson, A.B. and Williams, F.L.R. (2001) An Introductory Guide to Disease Mapping. John Wiley \& Son, Ltd., Chichester. http://dx.doi.org/10.1002/0470842571

[18] Aziz, S., Ngui, R., Lim, Y.A.L., et al. (2012) Spatial Pattern of 2009 Dengue Distribution in Kuala Lumpur Using GIS Application. Tropical Biomedicine, 29, 113-120. 